\title{
Enhanced magneto-optical Faraday effect in two-dimensional magnetoplasmonic structures caused by orthogonal plasmonic oscillations
}

\author{
D. M. Krichevsky $\odot,{ }^{1,3, *}$ A. N. Kalish $\odot,{ }^{2,3}$ M. A. Kozhaev,${ }^{3,4}$ D. A. Sylgacheva $\odot,{ }^{2,3}$ A. N. Kuzmichev, ${ }^{3}$ S. A. Dagesyan, ${ }^{2}$ \\ V. G. Achanta, ${ }^{5}$ E. Popova, ${ }^{6}$ N. Keller, ${ }^{6}$ and V. I. Belotelov ${ }^{2,3}$ \\ ${ }^{1}$ Center for Photonics and 2D Materials, Moscow Institute of Physics and Technology (National Research University), \\ 141700 Dolgoprudny, Russia \\ ${ }^{2}$ Lomonosov Moscow State University, 119991 Moscow, Russia \\ ${ }^{3}$ Russian Quantum Center, 121353 Moscow, Russia \\ ${ }^{4}$ Prokhorov General Physics Institute of the Russian Academy of Sciences, 119991 Moscow, Russia \\ ${ }^{5}$ Tata Institute of Fundamental Research, Homi Bhabha Road, Navy Nagar, Colaba, 400005 Mumbai, India \\ ${ }^{6}$ Groupe d'Etude de la Matière Condensée (GEMaC), CNRS-UVSQ, Université Paris-Saclay, 78035 Versailles, France
}

(Received 7 May 2020; revised 16 September 2020; accepted 17 September 2020; published 6 October 2020)

\begin{abstract}
We report the enhancement of the magneto-optical Faraday effect in a two-dimensional magnetoplasmonic grating with a square lattice. The structure consists of dielectric magnetic Bi-substituted iron garnet film with low thickness $(100 \mathrm{~nm})$ coated by a perforated gold layer. Enhancement of the Faraday rotation by about 2 times is shown experimentally and theoretically despite the suppression of the waveguiding modes. This happens primarily through excitation of surface plasmon polaritons propagating at the Au-garnet interface in two orthogonal directions along lattice vectors of the plasmonic grating.
\end{abstract}

DOI: 10.1103/PhysRevB.102.144408

\section{INTRODUCTION}

Among different optical effects, magneto-optics plays a crucial role due to its ability to control the polarization and intensity of optical radiation in a wide range of frequencies. The magneto-optical Faraday effect causes the rotation of the polarization plane of the light transmitted through magnetooptical materials. This feature is applied in optical isolators, polarizers, and sensors which are important in a large variety of technical systems [1-4]. Faraday rotators are, basically, bulky due to the weak Faraday effect in available magnetooptical materials. Together with a purely chemical approach to enhance magneto-optical effects, that is the synthesis of new gyrotropic materials, nanofabrication technologies open new prospects in magneto-optics.

Metallic and dielectric nanostructured materials, such as photonic crystals [5], plasmonic gratings [6], and metasurfaces [7] are important for modern photonic devices. Their combination with gyrotropic materials can significantly enhance magneto-optical effects. In metal-dielectric structures, magneto-optical effects are increased through excitation of photonic and plasmonic modes in the structure, such as surface plasmon polaritons (SPPs) [8-11], localized surface plasmons (LSPs) [12,13], and waveguide (WG) modes $[14,15]$. Moreover, in strong magnetic fields transmission and reflection of plasmonic gratings made of pure noble metals vary significantly [16]. For a metal-dielectric system in which a magnetic layer is thin enough so that WG modes propagation is restricted, the Faraday effect can be enhanced only

*krichevskii.dm@mipt.ru through SPP modes or LSP resonance excitation [17-19]. In structures containing plasmonic nanoparticles, gyrotropy induces orthogonal localized oscillations that provide resonant enhancement of the Faraday rotation [20]. Regarding surface propagating waves, we propose a similar mechanism for the Faraday effect enhancement, namely, the excitation of SPPs propagating in orthogonal directions.

In this paper, we report both experimental and theoretical studies of transmission and the Faraday rotation of twodimensional (2D) magnetoplasmonic systems based on the perforated metallic layer and the magnetic dielectric layer. Perforation of the plasmonic metal film with a periodic array of holes (2D periodic system) and the presence of a thin gyrotropic medium allowed us to excite orthogonal plasmons, i.e., two plasmonic modes propagating in orthogonal directions $[O X$ and $O Y$ directions as shown in Fig. 1(a)] at the metal-dielectric interface by normally incident light. It is shown that in such system orthogonal plasmonic oscillations at the interface between $\mathrm{Au}$ and iron garnet layers play a primary role in the enhancement of the Faraday rotation.

\section{METHODS}

\section{A. Sample fabrication}

The scheme of the studied structure is shown in Fig. 1(a). The structure consists of the Au layer of 80-nm thickness perforated with circular holes $(225 \mathrm{~nm})$ with a period of 570 (sample A) and $670 \mathrm{~nm}$ (sample B), a Bi-substituted iron garnet (BIG), in particular $\mathrm{Bi}_{3} \mathrm{Fe}_{5} \mathrm{O}_{12}$, layer of $100-\mathrm{nm}$ thickness, and gadolinium gallium garnet (GGG) substrate. The BIG film was grown on GGG (001) substrate by pulsed 
(a)

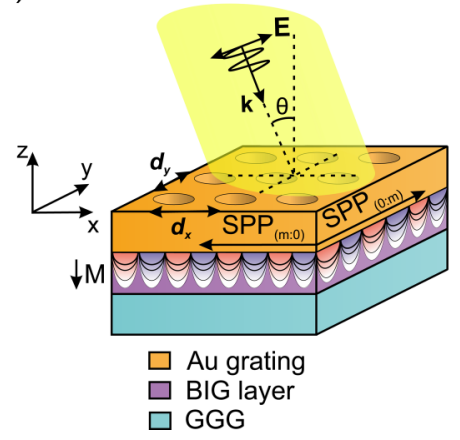

(b)

(c)

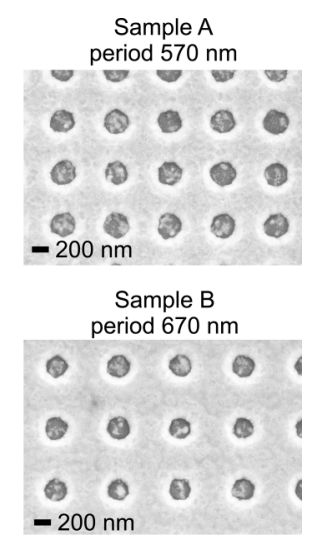

FIG. 1. Schematic representation of the examined structure (a) and SEM image of the fabricated magnetoplasmonic samples A (b) and $\mathrm{B}(\mathrm{c})$.

laser deposition [21]. The metal gratings of the experimentally studied samples were fabricated by electron-beam lithography of the thermally deposited gold layer. The scanning electron microscope (SEM) images of the fabricated samples is presented in Figs. 1(b) and 1(c). The holes' diameter estimated from the SEM image is approximately $225 \mathrm{~nm}$ and period is equal along $O X$ and $O Y$ directions $\left(d_{x}=d_{y}\right)$.

The incidence plane is $X Z$ and the light is TM-polarized. At normal incidence, the electric-field vector was directed along the $O X$ axis. For angle-dependent measurements and simulations, the polar angle $(\theta)$ is varied from $0^{\circ}$ to $30^{\circ}$.

\section{B. Optical and magneto-optical measurements}

Transmission dependence of the magnetoplasmonic gratings on the light wavelength and incidence angle was measured using the Fourier technique by the following experimental setup. The light emitted by the tungsten halogen lamp passed through the fiber. The output end of optical fiber with a diameter of $200 \mu \mathrm{m}$ was used as a pointlike light source. The light was collimated with an achromatic $75-\mathrm{mm}$ lens, polarized with a Glan-Taylor prism, and focused onto the sample with an additional achromatic 35-mm lens. After the sample, the light was collimated with a $20 \times$ microscope objective. Then light passed through a telescope made of 300- and 100-mm focal length lenses and was detected with a spectrometer. A spectrally and angularly decomposed light signal was detected by the spectrometer with a charge-coupled device camera.

The magneto-optical Faraday rotation spectra at fixed incidence angle was measured using another experimental setup. The light emitted by the tungsten-halogen was focused by a $75-\mathrm{mm}$ lens on a $100-\mu \mathrm{m}$-diameter pinhole to obtain a pointlike light source. Then the light was collimated with an achromatic $150-\mathrm{mm}$ lens, polarized with a Glan-Taylor prism, and focused onto the sample with an additional $300-\mathrm{mm}$ lens. The sample was placed inside an electromagnet which creates a normal to the sample plane magnetic field with a magnetic flux density of up to $3000 \mathrm{G}$. After the sample, the light was collimated with a 300-mm lens. Then light passed through an analyzer that is Glan-Taylor prism rotated by $45^{\circ}$ relative

to the previous polarizer. Finally, the light was focused on the spectrometer slit by a $100-\mathrm{mm}$ lens and detected with a charge-coupled device camera. To find the Faraday rotation, we use the following formula: $\varphi=\frac{1}{2} \sin ^{-1} \frac{I_{+}-I_{-}}{I_{+}+I_{-}}$, where $I_{+}$and $I_{-}$are intensities of light passing through the system for the positive and negative directions of the magnetization of the sample, correspondingly.

\section{Simulations}

The existence of orthogonal plasmonic modes at the metaldielectric interface and the mechanism of the Faraday rotation enhancement was verified by numerical solution of Maxwell equations using the rigorous coupled-wave analysis approach $[22,23]$.

The magnetization of the magnetic layer can be formulated in terms of the off-diagonal elements of complex permittivity tensor. In case of the Faraday configuration (magnetization is perpendicular to the structure's plane) the permittivity tensor of BIG is written as follows:

$$
\hat{\varepsilon}=\left(\begin{array}{ccc}
\varepsilon_{\mathrm{BIG}} & -i g & 0 \\
i g & \varepsilon_{\mathrm{BIG}} & 0 \\
0 & 0 & \varepsilon_{\mathrm{BIG}}
\end{array}\right) .
$$

For simulations the dispersion of $\varepsilon_{\mathrm{BIG}}$ was taken into account, so that $\varepsilon_{\mathrm{BIG}}=6.37+0.11 i$ for the wavelength of $850 \mathrm{~nm}$. The gyration $g$ of the BIG layer was kept constant: $g=(1-0.015 i) 10^{-2}$. Gyration of BIGs is typically dispersive, however in the region of interest its deviation is quite small and average value typical for such kind of iron garnets can be utilized in calculations [24]. Optical constants of Au in the region of interest were taken from Ref. [25]. The refractive index of GGG was equal to 1.97 .

In numerical calculations the Faraday rotation angle was calculated using following relation:

$$
\varphi=\operatorname{Re}\left(\tan ^{-1} \frac{E_{y}}{E_{x}}\right) \frac{180^{\circ}}{\pi},
$$

where $E_{x, y}$ are absolute values of $x$ and $y$ electric fields components in the zeroth diffraction order. For calculation of zero-order transmittance in the simulation we used the following formula:

$$
T_{0}=\frac{\left(\left|E_{x}\right|^{2}+\left|E_{y}\right|^{2}+\left|E_{z}\right|^{2}\right)}{\left|E_{0}\right|^{2}} \frac{\operatorname{Re}\left(\sqrt{n_{\text {substrate }}^{2}-\cos ^{2} \theta}\right)}{\cos \theta} .
$$

Here $\left|E_{x, y, z}\right|$ are absolute values of corresponding electric fields components in the zeroth diffraction order, $\left|E_{0}\right|$ is an absolute value of the incident light electric-field amplitude, $\theta$ is an angle of incidence (see Fig. 1), and $n_{\text {substrate }}$ is GGG refractive index.

\section{RESULTS AND DISCUSSION}

\section{A. Photonic/plasmonic mode description}

The transmission spectra of the Au/BIG/GGG structure illuminated by linearly polarized light $(E$ field is directed along $O X$ axis) exhibit several resonant dips in the range of interest which correspond to the excitation of the optical modes in the structure. As it is depicted in Fig. 2 there are well-defined 
(a)

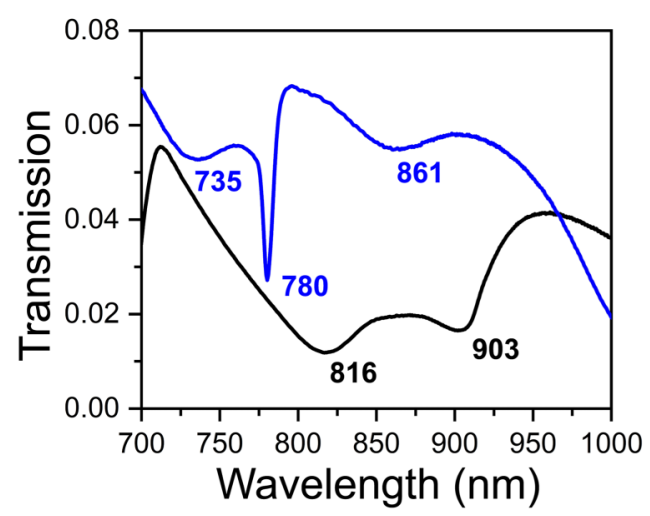

(c)

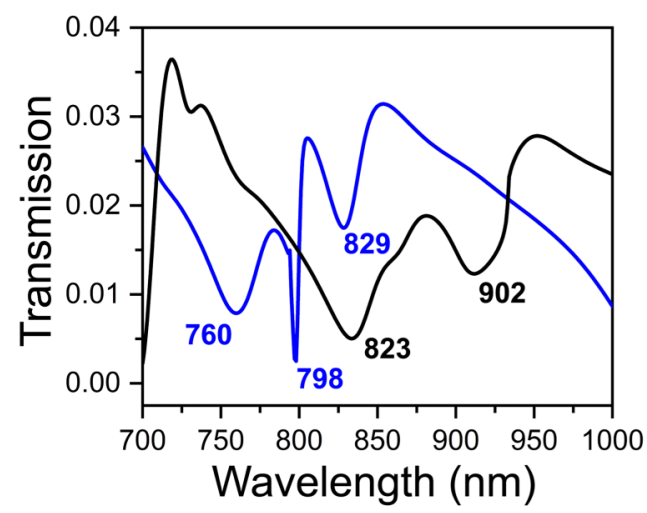

- Sample A (period $570 \mathrm{~nm})$ (b)

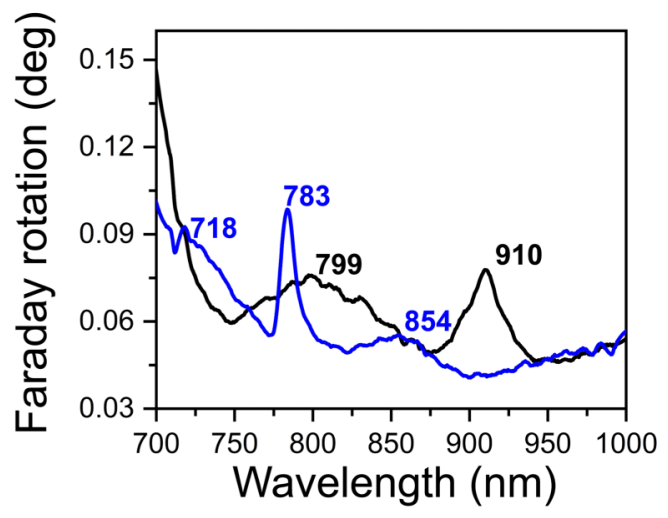

(d)

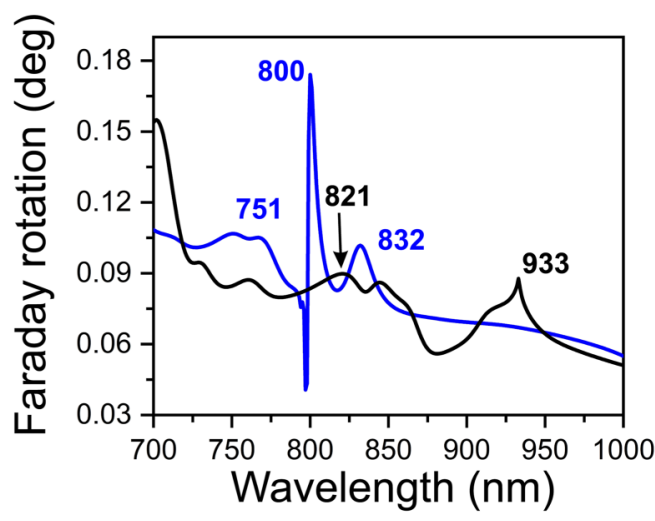

Sample B (period $670 \mathrm{~nm}$ )

FIG. 2. Experimental (a), (b) and simulated (c), (d) transmission and the Faraday rotation spectra of the studied samples. Black and blue curves represent sample A and B, correspondingly.

resonances at 735,780 , and $861 \mathrm{~nm}$ in transmission and the Faraday rotation experimental spectra of sample A. Similar resonances for sample B are observed evolving as the structure period increases. However, the high-quality resonance at $780 \mathrm{~nm}$ is not observed in the spectrum of sample B. As it will be shown further this resonance is overlapped with the resonance at $903 \mathrm{~nm}$. Maximum Faraday rotation of $0.1^{\circ}$ and $0.06^{\circ}$ for sample A was found at 783 and $854 \mathrm{~nm}$, which are 1.6 and 1.25 times larger than for the bare BIG film. The highest value of the Faraday rotation enhancement factor ( 2 times) in comparison to the bare BIG film was found for sample B at $910 \mathrm{~nm}$. The simulated results correlate with the experimental results, however some mismatch is present which can be caused by inhomogeneity of the fabricated samples. As it will be explained further the observed resonant Faraday rotation enhancement corresponds to excitation of different optical modes, including diagonal and off-diagonal SPPs. To explain the mentioned features in the experimental and simulated spectra, let us consider behavior of the optical modes.

Optical modes of the structure can be excited due to diffraction on the grating according to the phase-matching condition:

$$
\beta^{2}=\left(k_{0} \sin (\theta)+2 \pi \frac{m}{d_{x}}\right)^{2}+\left(2 \pi \frac{n}{d_{y}}\right)^{2}
$$

where $\beta$ is the propagation constant of a mode (either SPP or WG mode), $k_{0}$ is the vacuum wave number, $\theta$ is the incident angle, $d_{x}$ and $d_{y}$ are periods along $x$ and $y$ directions, respectively, $m$ and $n$ are integers which correspond to the SPP order. As it was mentioned earlier in our samples $d_{x}=d_{y}$. At normal incidence of light the propagation constant depends only on the period of the hole spacing and integer numbers $m$ and $n$. In this case the SPP can propagate along $O X$ axis $(m \neq 0$ and $n=0)$, along $O Y$ axis $(n \neq 0$ and $m=0)$ and at a certain angle to the $O X$ and $O Y$ axes $(n, m \neq 0)$. The latter case will be called diagonal modes. In the first two cases propagation constants can take equal values. This case corresponds to orthogonal propagation of SPPs.

For perforated metal films surrounded by two semi-infinite dielectric media, the positions of the transmission dips are well described by the condition of the SPP excitation by light of wavelength $\lambda$ [26]. In the case of nonsymmetric dielectric environment the propagation constants of coupled SPP modes on the top and bottom metal-dielectric interfaces can be found using transcendental equation [27]:

$$
\tanh \left(\gamma_{2} h_{1}\right)\left(\varepsilon_{1} \varepsilon_{3} \gamma_{2}^{2}+\varepsilon_{2}{ }^{2} \gamma_{1} \gamma_{3}\right)+\left[\gamma_{2}\left(\varepsilon_{1} \gamma_{3}+\varepsilon_{3} \gamma_{1}\right) \varepsilon_{2}\right]=0
$$

where $h_{1}$ is the thickness of the metal film, $\gamma_{1}^{2}=\beta^{2}-$ $\varepsilon_{1} k_{0}^{2} ; \gamma_{2}^{2}=\beta^{2}-\varepsilon_{2} k_{0}^{2} ; \gamma_{3}^{2}=\beta^{2}-\varepsilon_{3} k_{0}^{2}$, indexes 1 and 3 denote the top and bottom dielectric environment, respectively, while 2 indicates metal. When the film thickness is 


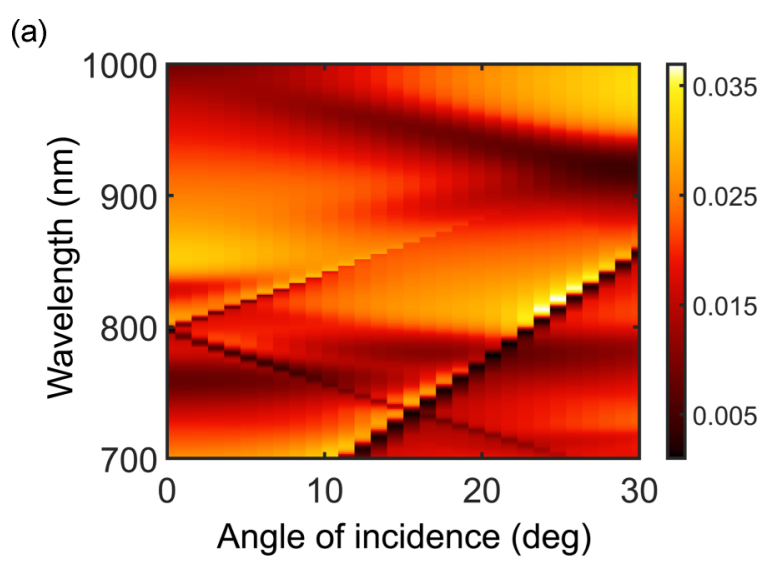

(c)

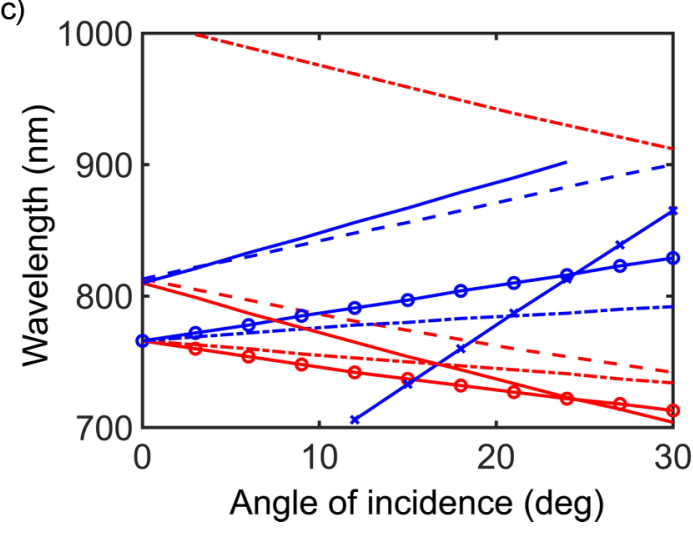

(b)

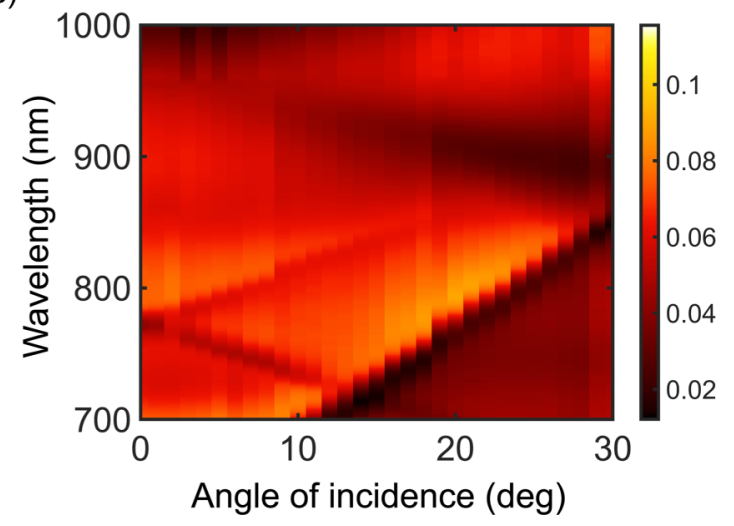

FIG. 3. Numerically calculated (a), experimentally measured (b) transmission and modes' dispersions (c) as a function of wavelength (vertical axis) and the incident angle (horizontal axis) for sample A. $m$ and $n$ are integers in phase-matching conditions [Eq. (3)].

much greater than the skin depth, Eq. (2) can be reduced to

$$
\left(\varepsilon_{2} \gamma_{1}+\varepsilon_{1} \gamma_{2}\right)\left(\varepsilon_{2} \gamma_{3}+\varepsilon_{3} \gamma_{2}\right)=0
$$

The solutions of Eq. (5) describe decoupled SPPs which propagate along the $\varepsilon_{2}-\varepsilon_{1}$ and $\varepsilon_{2}-\varepsilon_{3}$ interfaces with dispersion relation:

$$
\begin{aligned}
& \beta_{1}(\infty)=\varepsilon_{1}{ }^{1 / 2} k_{0}\left[\frac{\varepsilon_{2}}{\varepsilon_{2}+\varepsilon_{1}}\right]^{1 / 2}, \\
& \beta_{3}(\infty)=\varepsilon_{3}{ }^{1 / 2} k_{0}\left[\frac{\varepsilon_{2}}{\varepsilon_{2}+\varepsilon_{3}}\right]^{1 / 2} .
\end{aligned}
$$

Spectral positions of the WG modes propagating inside the dielectric layer can be obtained using the transcendental equation for fundamental TM and TE regimes [28]:

$$
\begin{gathered}
\text { TM case }: \tan \left(h_{2} k_{3}\right)=\frac{\left(\varepsilon_{4} \gamma_{2}+\varepsilon_{2} \gamma_{4}\right) \varepsilon_{3} k_{3}}{\varepsilon_{2} \varepsilon_{4} k_{3}^{2}-\varepsilon_{3}^{2} \gamma_{2} \gamma_{4}}, \\
\text { TE case }: \tan \left(h_{2} k_{3}\right)=\frac{\left(\gamma_{2}+\gamma_{4}\right) k_{3}}{k_{3}^{2}-\gamma_{2} \gamma_{4}},
\end{gathered}
$$

where $h_{2}$ is the thickness of the BIG layer, $\varepsilon_{3}$ is the dielectric permittivity of BIG, $\varepsilon_{4}$ is the dielectric permittivity of the GGG substrate, and

$$
\begin{aligned}
& k_{3}^{2}=\varepsilon_{3} k_{0}{ }^{2}-\beta^{2}, \\
& \gamma_{4}^{2}=\beta^{2}-\varepsilon_{4} k_{0}{ }^{2} .
\end{aligned}
$$

An effective index of the mode $n_{\text {eff }}=\operatorname{Re}\left(\frac{\beta}{k_{0}}\right)$ gives essential information about the nature of the excited mode. For the SPP modes the effective index should be higher than the refractive index of the magnetic film. For the photonic WG mode the effective index should satisfy the following inequality: $n_{\text {BIG }}>n_{\text {eff }}>n_{\text {substrate }}$, where $n_{\text {BIG }}=\operatorname{Re} \sqrt{\varepsilon_{3}}$ is the refractive index of BIG.

Guided by these concepts the dispersion curves were calculated [Fig. 3(c)] and compared with experimentally measured and numerically calculated transmission as a function of wavelength and incident angle [Figs. 3(a) and 3(b)]. Noticeably, there are rather small discrepancies between calculated positions of the resonances and the one obtained from experimental data both in transmission as well as the Faraday rotation spectra. There are a few origins of these features. The first one is connected with fabrication inaccuracies resulting in a little difference between geometrical properties (such as period of the gratings, holes diameter, etc.) of the experimental samples with their calculated counterparts. The second one is associated with the nature of the observed resonances which are asymmetric Fano type. Moreover, to take into account the presence of the holes in the Au layer, the dielectric permittivity of the magnetic layer was varied to obtain the best correspondence between the experimentally observed and theoretically calculated spectral positions of optical resonances for all samples. However, some mismatch still took place due to the "empty lattice" approach [29]. 
TABLE I. Spectral positions of the resonances in transmission spectra of the samples and corresponding optical modes for normal incidence of linearly polarized light.

\begin{tabular}{|c|c|c|c|c|c|}
\hline \multirow[t]{2}{*}{ A } & $\begin{array}{l}m= \pm 1 n= \pm 2 \\
m= \pm 2 n= \pm 1\end{array}$ & & 735 & 760 & 766 \\
\hline & $m= \pm 2 n=0$ & & 861 & 823 & 813 \\
\hline \multirow{2}{*}{ B } & $m= \pm 2 n=0$ & & 903 & 902 & 875 \\
\hline & & $m= \pm 1 n= \pm 1$ & 910 & 933 & 934 \\
\hline
\end{tabular}

Sharp resonances in transmission and the Faraday rotation angle spectra (Fig. 2) correspond to the excitation of the quasiTE WG modes. The Faraday effect enhancement due to the WG mode excitation that can be observed in similar structures was previously reported $[14,15]$. Special attention should be paid to the SPP-assisted Faraday effect enhancement. For normally incident light, spectral positions of the resonances and corresponding optical modes are presented in Table I.

Resonances where the SPP modes, both diagonal ( $m$ and $n$ are nonzero arbitrary) and off diagonal ( $m$ is an arbitrary integer, while $n=0$ and vice versa), are excited attract the greatest interest. The nature of the enhanced Faraday rotation on these modes is identical for the two studied structures. Here we focus on off-diagonal one, namely $\mathrm{SPP}_{ \pm 2: 0}$ which is observed at $\sim 823 \mathrm{~nm}$ in the numerically simulated transmission spectrum of sample A. Orthogonal SPP modes cannot be excited at the interface between isotropic dielectric and metal by light with polarization vector directed along periodicity direction of the plasmonic grating. Consequently, gyrotropy-induced orthogonal SPPs excitation and the stimulated Faraday rotation can be clearly analyzed and visualized from the electromagnetic field patterns. Let us consider the case of off-diagonal SPP modes thoroughly.

\section{B. Mechanism of the Faraday rotation enhancement and orthogonal SPPs}

First, consider the case of orthogonal SPPs excitation on the boundary between nonmagnetic dielectric layer and periodically perforated metal. According to phase-matching conditions [Eq. (3)] at normal incidence of the light if the hole pattern period of the metal layer is equal $\left(d_{x}=d_{y}\right)$ SPPs with $\left(m=m_{0}, n=0\right)$ and $\left(m=0, n=m_{0}\right)$, where $m_{0}$ is a nonzero arbitrary integer, can be excited at an equal wavelength. However, if polarization of the incident light is directed along the $O X$ axis, only one SPP with $\left(m=m_{0}, n=0\right)$ can excited because polarization of the second SPP with $\left(m=0, n=m_{0}\right)$ is orthogonal to polarization of the incident light. Nevertheless, if polarization of the SPP-exciting light is rotated through the Faraday effect or by external optical devices, it is possible to launch two SPPs moving in orthogonal directions. These SPPs will propagate along the $O X$ and $O Y$ axes, respectively.

To illustrate this idea a simulation was performed. According to the numerical estimations, $\mathrm{SPP}_{2: 0}$ is excited at $\sim 823 \mathrm{~nm}$ in sample A by normally incident light exposure. When materials are isotropic and the structure is illuminated by linearly polarized light only $E_{x}, E_{z}, H_{y}$ components exist and the $\mathrm{SPP}_{2: 0}$ propagates along the $O X$ axis. It should be noted that, for instance, the $H_{y}$ component of the $\mathrm{SPP}_{2: 0}$ is distributed inhomogeneously in the $y-z$ plane while in the $x-z$ plane it resembles a standing wave concentrated at the Au-BIG interface. Polarization rotation of the incident light by $45^{\circ}$ excites additional surface wave $\mathrm{SPP}_{0: 2}$ with the field components $H_{x}, H_{z}, E_{y}$ (see Fig. 4) which propagates along the $O Y$ axis. The amplitudes of both $\mathrm{SPP}_{2: 0}$ and $\mathrm{SPP}_{0: 2}$ fields are also equal due to the symmetry of the structure and excitation conditions.

A similar effect can be observed for linearly polarized light illumination polarized along the $O X$ axis when permittivity of the dielectric magnetic layer is gyrotropic and the external magnetic field is applied in the polar configuration. The magnetization of the BIG layer induces additional field components $\left(E_{y}, H_{x}, H_{z}\right)$ of the initial $\mathrm{SPP}_{2: 0}$ [17]. As a result, these stimulated components generate the orthogonal $\mathrm{SPP}_{0: 2}$ mode propagating along the $O Y$ direction.

At the excitation wavelength for $\mathrm{SPP}_{2: 0} H_{x}$ and $H_{y}$ components exist in the field distribution for both $\mathrm{SPP}_{2: 0}$ and $\mathrm{SPP}_{0: 2}$ as it is depicted in Fig. 5. Due to the gyrotropy of the BIG layer TM-polarized $\mathrm{SPP}_{2: 0}$ acquires additional TE components with amplitude proportional to $g$. These components exist only in the $x-z$ plane of the field patterns. Stimulated by this phenomenon orthogonal $\mathrm{SPP}_{0: 2}$ is launched. As it can be noticed from comparison of Fig. 5 and Fig. 4 the resulting TE-components field distribution in $y-z$ plane are mainly determined by the orthogonal $\mathrm{SPP}_{0: 2}$. The induced $E_{y}$ component of these coupled plasmonic oscillations scatters into the far field and produces an additional resonant contribution to the Faraday rotation. As a result overall Faraday rotation enhancement is caused by orthogonal $\mathrm{SPP}_{0: 2}$. A similar effect was observed in Ref. [30] for longitudinal Kerr configuration. An increased magneto-optical activity stimulated by SPPs excitation was found for both TM and TE polarizations of the incident light. The enhancement of polarization rotation was also observed for both diagonal and off-diagonal SPPs.

On the other hand, according to Eq. (3) if periods in $O X$ and $O Y$ directions are different the phase-matching conditions forbid simultaneous excitation of the orthogonal SPP modes. As a consequence, only $E_{y}, H_{x}, H_{z}$ components arising due to 

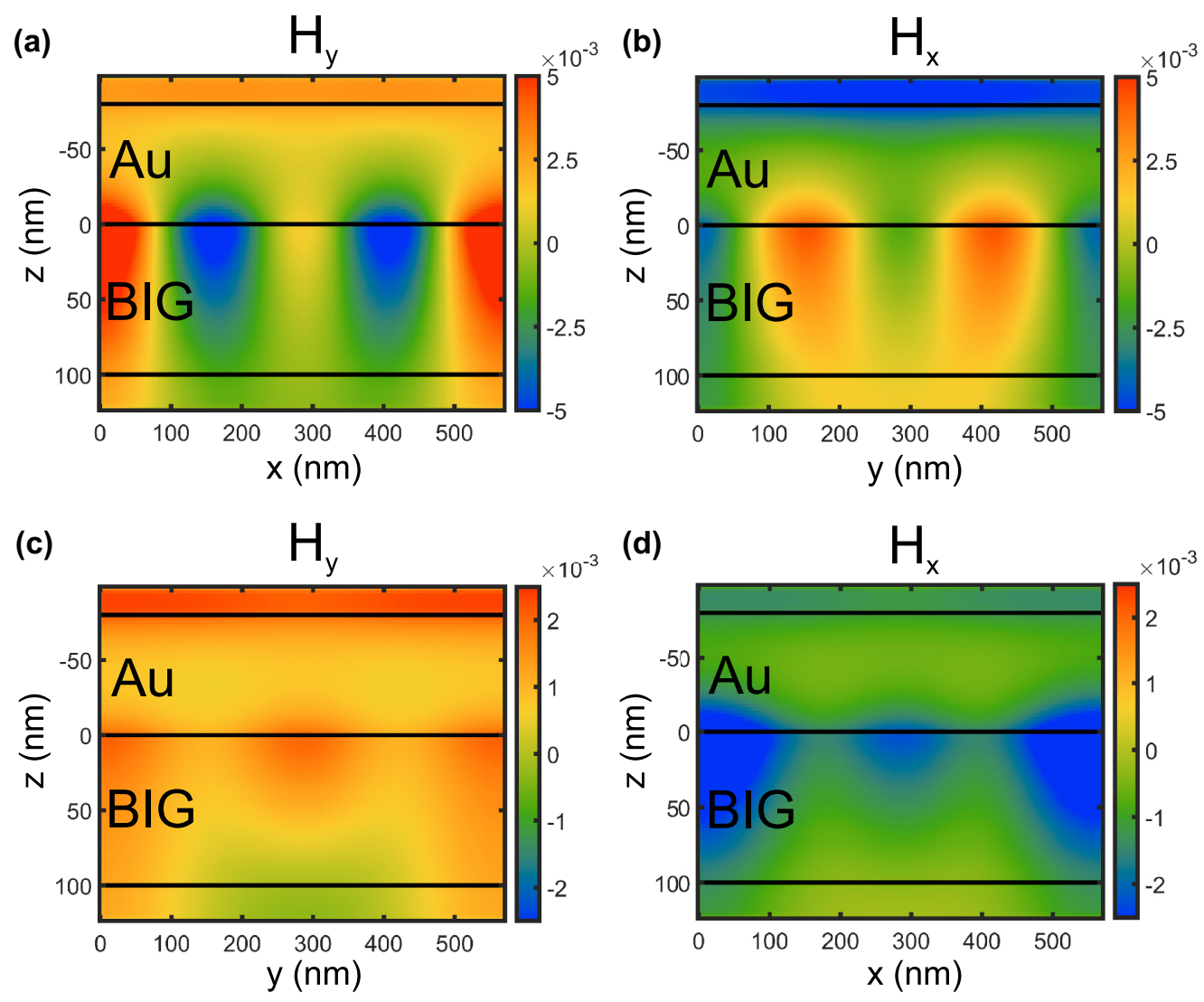

FIG. 4. Simulated field distribution of $H_{y}$ (a), (c) and $H_{x}$ (b), (d) components in $x-z$ and $y$ - $z$ planes at 823 nm for zero gyrotropy for sample A. The resonances are excited by normally incident light with polarization rotated by $45^{\circ}$ relative to $O X$ axis.

the gyrotropy of the magnetic layer impact on the Faraday rotation. In our simulation, we changed $d_{y}$ to confirm this statement so that $d_{y}=1.3 d_{x}$. In this case, only $\mathrm{SPP}_{2: 0}$ can be excited by normal incident light illumination at $\sim 830 \mathrm{~nm}$ while the orthogonal one has different wavelength. The transmission spectra for this case are presented in Fig. 6(a). As a result, the Faraday rotation at $830 \mathrm{~nm}$ is weaker [Fig. 6(b)] and the enhancement factor reaches 1.23 while for sample A with equal periods this value is 1.6. Thus, the excitation of the orthogonal SPPs is an efficient tool for the Faraday rotation increase.

Besides the Faraday rotation enhancement through orthogonal SPPs excitation an interesting peculiarity is observed for sample B. In the region of 910-930 nm the orthogonal SPP band overlaps with the quasi-TE WG mode (Fig. 2, black curves). As a result of these two modes coupling a greater enhancement of the Faraday angle rotation ( $\sim 2$ times) is produced. A similar coupling mechanism between the TM SPP and TE WG modes was investigated earlier in Ref. [31]. This feature can be utilized in hybrid nanostructures that support both the quasi-TE WG mode excitation and orthogonal SPPs, such as photonic/plasmonic integrated circuits.

It should be noted that the efficiency of higher-order SPP excitation is lower than for the first order. Consequently, the Faraday rotation enhancement can be additionally increased through the first-order SPP excitation. Moreover, low transmittance of the structures under consideration makes them less suitable for applications. To solve these issues we carried out auxiliary calculations. The simulated structure was similar to sample A, but the period was decreased to $320 \mathrm{~nm}$ and the holes diameter was increased to $300 \mathrm{~nm}$ (sample C). All other parameters were equivalent. Transmission and the Faraday rotation angle spectra of sample $\mathrm{C}$ are presented in Fig. 7 in comparison with the ones obtained for sample $\mathrm{A}$.

Transmission spectrum of sample C [Fig. 7(a) has a resonant dip at $893 \mathrm{~nm}$ that corresponds to the excitation of the orthogonal SPPs $( \pm 1: 0)$ and $(0: \pm 1)$. Transmittance reaches values of $\sim 15 \%$ which is almost an order of magnitude higher than for sample A. At the same resonant position the Faraday rotation enhancement is observed [Fig. 7(b)]. The enhancement reaches value of 2.26 caused by both boosted transparency and increased excitation efficiency. What is more important the ellipticity coefficient that can be calculated as $k=\tanh \left\{\operatorname{Im}\left[\tan ^{-1}\left(\frac{E_{y}}{E_{x}}\right)\right]\right\}$ for all studied samples approaches zero $\left(\sim 10^{-4}\right)$ which is a merit that make such structures attractive for applications.

There are many different ways to increase the transmission of plasmonic nanostructured materials. The simplest methods are decreasing the thickness of the highly absorbing layers which is Au layer in our case or increasing the diameter of the holes. In the first case, with increased transmittance the quality factor and excitation efficiency of the SPP resonances dramatically decrease. Most importantly, the amplification of the Faraday rotation angle can almost disappear. More efficient approach is to increase the hole size. As it was shown previously for sample $\mathrm{C}$ (where the relation between the hole 

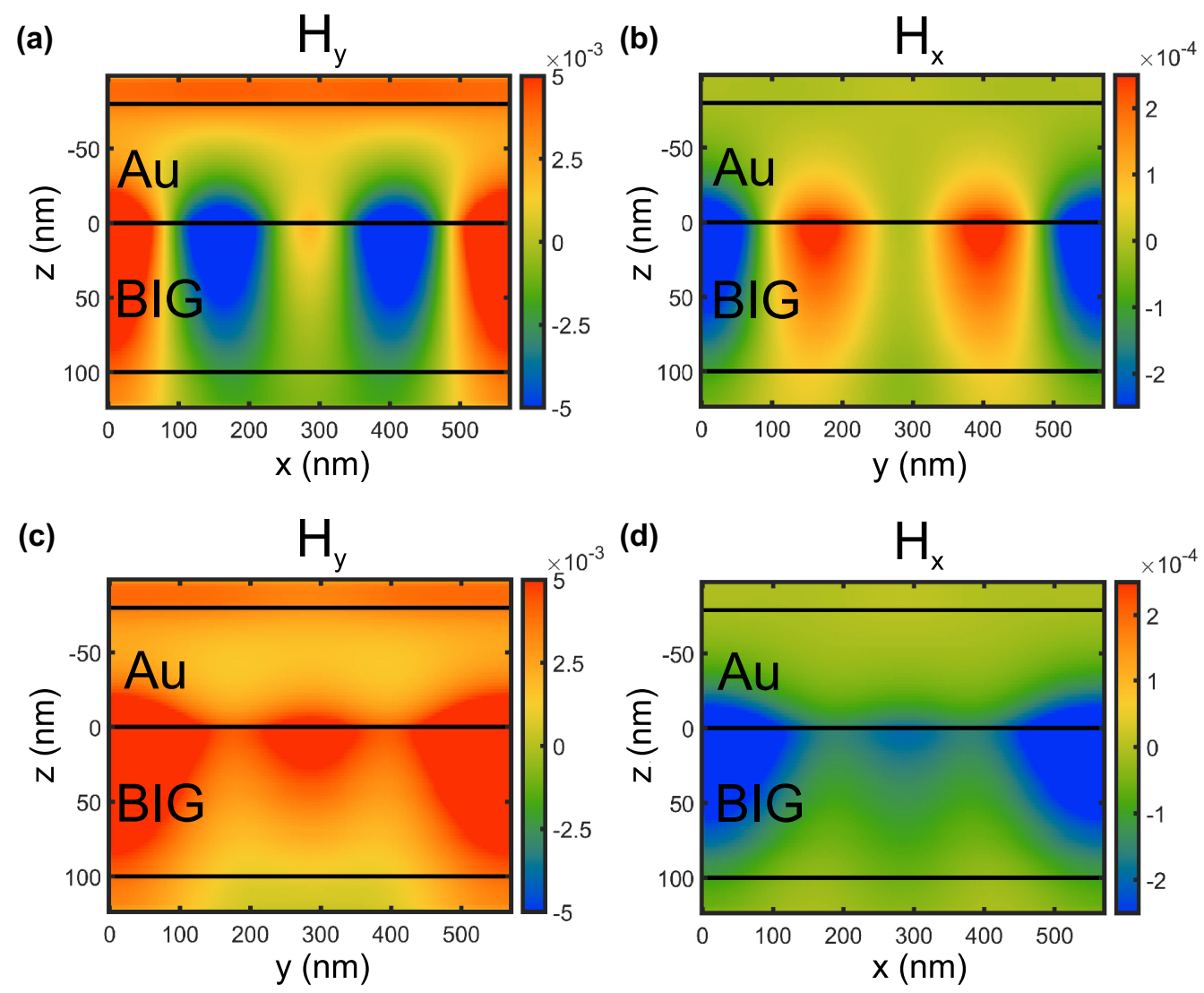

FIG. 5. Simulated field distribution of $H_{y}$ (a), (c) and $H_{x} H_{x}$ (b), (d) components in $x-z$ and $y$ - $z$ planes at 823 nm for nonzero gyrotropy for sample A.

diameter and period of the grating is $\sim 0.94$ ) transmittance can reach $15 \%$ which is far higher than the one for sample A. Moreover, other parameters of the structure influence the transmittance: dielectric properties of the media, period of the grating, operating frequency/wavelength. Proper selection of structure's parameters can lead to extraordinary transmission. Eventually, through tailoring geometrical and dielectric parameters of the system we can achieve higher values of transmittance (including effects of extraordinary transmission) and the Faraday rotation enhancement.

(a)

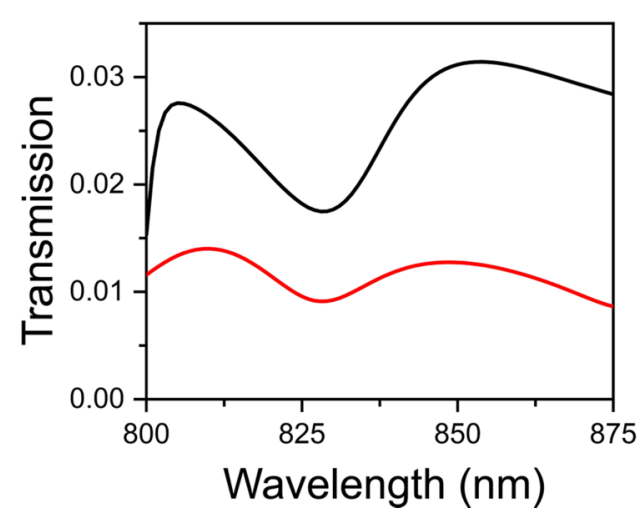

\section{CONCLUSION}

We proposed a mechanism of the Faraday effect enhancement in magnetoplasmonic structures. Namely, we have shown that the Faraday polarization rotation in the magnetoplasmonic structures with 2D periodicity supporting propagating SPPs can be enhanced by simultaneous excitation of the SPP modes propagating in orthogonal directions. This is due to the fact that gyrotropy induces electromagnetic field components of the initial SPP and auxiliary orthogonal one, in particular $E_{y}$, which scatter in the far field. We have illustrated

(b)

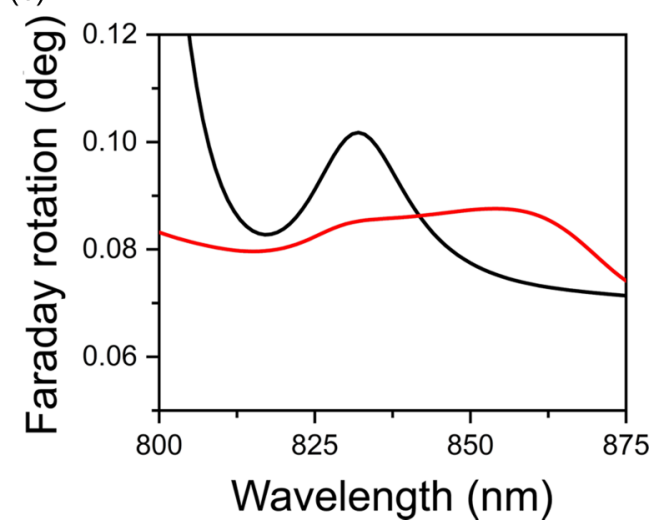

FIG. 6. Simulated transmission (a) and the Faraday rotation angle (b) of sample A with $d_{y}=d_{x}$ (black) and $d_{y}=1.3 d_{x}$ (red). 


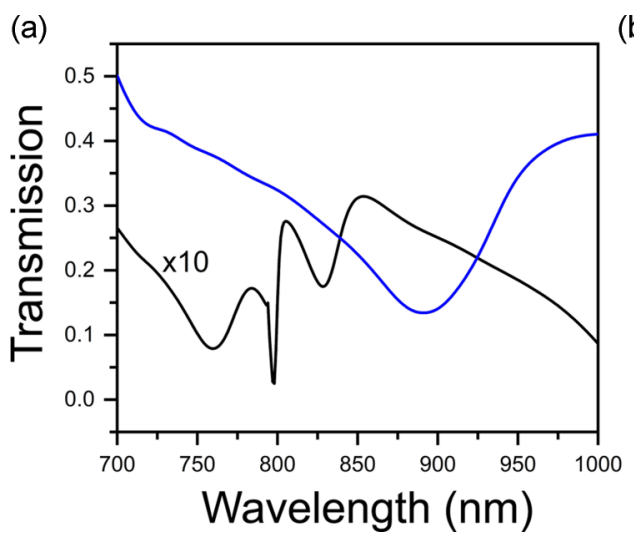

(b)

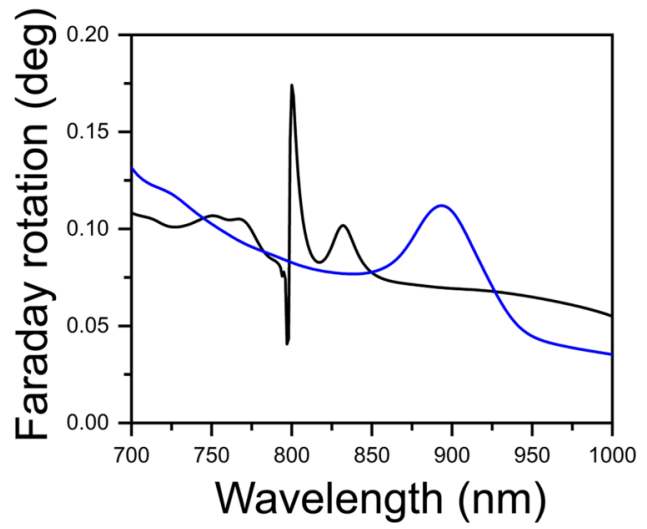

FIG. 7. Transmission (a) and the Faraday rotation (b) spectra of sample A (black) and C (blue). Transmission spectrum of sample A is multiplied by 10 .

this idea with a systematic analysis of the excited modes in a hybrid Au-BIG-GGG heterostructure and electromagnetic fields distributions. We have demonstrated 1.6 times enhancement of the Faraday effect caused by excitation of the coupled $\mathrm{SPP}_{2: 0}-\mathrm{SPP}_{0: 2}$ oscillations at the Au-BIG interface. The enhancement factor can be further increased when the excitation of the orthogonal SPPs is accompanied by excitation of quasiTE WG modes, so the two mechanisms for the enhancement are combined.

The proposed mechanism of the Faraday effect enhancement is of both fundamental and practical importance. On the one hand, it is an efficient tool involving propagating plasmonic modes, while conventionally the Faraday effect gets enhanced due to either localized or waveguide modes.
On the other hand, the obtained results open up possibilities for design of miniature devices based on thin films, since only surface modes are involved.

\section{ACKNOWLEDGMENTS}

This work was financially supported by the Russian Foundation for Basic Research (Project No. N 18-52-80038). V.G.A. acknowledges support from the Department of Science and Technology, India via Projects Number INT/RUS/RFBR/317, and Number DST/IMRCD/BRICS/PilotCal12/Optonanospin/2018 (G). The authors thank D. O. Ignatyeva for the fruitful discussion.
[1] H. Yoshida, K. Tsubakimoto, Y. Fujimoto, K. Mikami, H. Fujita, N. Miyanaga, H. Nozawa, H. Yagi, T. Yanagitani, Y. Nagata, and H. Kinoshita, Opt. Express 19, 15181 (2011).

[2] A. K. Zvezdin and V. A. Kotov, Modern Magnetooptics and Magnetooptical Materials (CRC Press, Boca Raton, FL, 1997).

[3] B. J. Stadler and T. Mizumoto, IEEE Photonics J. 6, 1 (2013).

[4] L. Sun, S. Jiang, and J. R. Marciante, Opt. Express 18, 5407 (2010).

[5] D. Marpaung, C. Roeloffzen, R. Heideman, A. Leinse, S. Sales, and J. Capmany, Laser Photonics Rev. 7, 506 (2013).

[6] D. K. Gramotnev and S. I. Bozhevolnyi, Nat. Photonics 4, 83 (2010).

[7] S. Jahani and Z. Jacob, Nat. Nanotechnol. 11, 23 (2016).

[8] V. I. Belotelov, I. A. Akimov, M. Pohl, V. A. Kotov, S. Kasture, A. S. Vengurlekar, A. V. Gopal, D. R. Yakovlev, A. K. Zvezdin, and M. Bayer, Nat. Nanotechnol. 6, 370 (2011).

[9] A. N. Kalish, R. S. Komarov, M. A. Kozhaev, V. G. Achanta, S. A. Dagesyan, A. N. Shaposhnikov, A. R. Prokopov, V. N. Berzhansky, A. K. Zvezdin, and V. I. Belotelov, Optica 5, 617 (2018).

[10] G. A. Knyazev, P. O. Kapralov, N. A. Gusev, A. N. Kalish, P. M. Vetoshko, S. A. Dagesyan, A. N. Shaposhnikov, A. R. Prokopov, V. N. Berzhansky, A. K. Zvezdin, and V. I. Belotelov, ACS Photonics 5, 4951 (2018).

[11] O. V. Borovkova, H. Hashim, M. A. Kozhaev, S. A. Dagesyan, A. Chakravarty, M. Levy, and V. I. Belotelov, Appl. Phys. Lett. 112, 063101 (2018).
[12] H. Uchida, Y. Masuda, R. Fujikawa, A. V. Baryshev, and M. Inoue, J. Magn. Magn. Mater. 321, 843 (2009).

[13] E. Almpanis, P. A. Pantazopoulos, N. Papanikolaou, V. Yannopapas, and N. Stefanou, J. Opt. Soc. Am. B 33, 2609 (2016).

[14] V. I. Belotelov, L. L. Doskolovich, and A. K. Zvezdin, Phys. Rev. Lett. 98, 077401 (2007).

[15] C. Lei, L. Chen, Z. Tang, D. Li, Z. Cheng, S. Tang, and Y. Du, Opt. Lett. 41, 729 (2016).

[16] Y. M. Strelniker and D. J. Bergman, Phys. Rev. B 59, R12763 (1999).

[17] A. N. Kalish, D. O. Ignatyeva, V. I. Belotelov, L. E. Kreilkamp, I. A. Akimov, A. V. Gopal, M. Bayer, and A. P. Sukhorukov, Laser Phys. 24, 094006 (2014).

[18] A. V. Baryshev and A. M. Merzlikin, J. Opt. Soc. Am. B 33, 1399 (2016).

[19] N. Maccaferri, A. Berger, S. Bonetti, V. Bonanni, M. Kataja, Q. H. Qin, S. van Dijken, Z. Pirzadeh, A. Dmitriev, J. Nogués, J. Åkerman, and P. Vavassori, Phys. Rev. Lett. 111, 167401 (2013).

[20] H. Y. Feng, F. Luo, R. Kekesi, D. Granados, D. Meneses-Rodríguez, J. M. García, A. García-Martín, G. Armelles, and A. Cebollada, Adv. Opt. Mater. 2, 612 (2014).

[21] E. Popova, M. Deb, L. Bocher, A. Gloter, O. Stéphan, B. WarotFonrose, B. Berini, Y. Dumont, and N. Keller, J. Appl. Phys. 121, 115304 (2017). 
[22] M. G. Moharam, E. B. Grann, D. A. Pommet, and T. K. Gaylord, J. Opt. Soc. Am. A 12, 1068 (1995).

[23] L. Li, J. Opt. A-Pure Appl. Opt. 5, 345 (2003).

[24] J. Y Chin et al., Nat. Commun. 4, 1 (2013).

[25] P. B. Johnson and R. W. Christy, Phys. Rev. B 6, 4370 (1972).

[26] T. W. Ebbesen, H. J. Lezec, H. F. Ghaemi, T. Thio, and P. A. Wolff, Nature (London) 391, 667 (1998).

[27] J. J. Burke, G. I. Stegeman, and T. Tamir, Phys. Rev. B 33, 5186 (1986).
[28] M. J. Adams, An Introduction to Optical Waveguides (Wiley, New York, 1981), Vol. 14.

[29] S. G. Tikhodeev, A. L. Yablonskii, E. A. Muljarov, N. A. Gippius, and T. Ishihara, Phys. Rev. B 66, 045102 (2002).

[30] N. Maccaferri, X. Inchausti, A. García-Martín, J. C. Cuevas, D. Tripathy, A. O. Adeyeye, and P. Vavassori, ACS Photonics 2, 1769 (2015).

[31] D. Floess, J. Y. Chin, A. Kawatani, D. Dregely, H.-U. Habermeier, T. Weiss, and H. Giessen, Light Sci. Appl. 4, e284 (2015). 